\title{
On a condition for type-I Bose-Einstein condensation in random potentials in $d$ dimensions
}

\author{
Joachim Kerner 1 \\ Department of Mathematics and Computer Science \\ FernUniversitt in Hagen \\ 58084 Hagen \\ Germany \\ Maximilian Pechmann 2 \\ Department of Mathematics \\ University of Tennessee \\ Knoxville, TN 37996 \\ USA \\ Wolfgang Spitzer 3 \\ Department of Mathematics and Computer Science \\ FernUniversitt in Hagen \\ 58084 Hagen \\ Germany
}

\begin{abstract}
In this paper we discuss Bose-Einstein condensation (BEC) in systems of pairwise non-interacting bosons in random potentials in $d$ dimensions. Working in a rather general framework, we provide a "gap condition" which is sufficient to conclude existence of type-I BEC in probability and in the $r$ th mean. We illustrate our results in the context of the well-known (one-dimensional) Luttinger-Sy model. Here, whenever the particle density exceeds a critical value, we show in addition that only the ground state is macroscopically occupied.
\end{abstract}

\footnotetext{
${ }^{1}$ E-mail address: joachim.kerner@fernuni-hagen.de

${ }^{2}$ E-mail address: mpechmann@utk.edu

${ }^{3}$ E-mail address: wolfgang.spitzer@fernuni-hagen.de
} 


\section{Introduction}

Bose-Einstein condensation (BEC) is usually referred to as a macroscopic occupation of a one-particle state. Instead, a broader definition of BEC speaks of generalized BoseEinstein condensation (g-BEC) where only a macroscopic occupation of an arbitrarily small energy band of one-particle states is required. Depending on the quantity of macroscopically occupied one-particle states in the condensate one then distinguishes three types of $\mathrm{g}$ BEC: A condensation of type I is said to occur if the number of macroscopically occupied one-particle states is finite but at least one. If there are infinitely many such one-particle states, the condensation is said to be of type II. Lastly, a generalized condensate in which none of the one-particle states are macroscopically occupied is called a type-III condensate.

Although it is generally believed that repulsive interactions between the particles should be taken into account when considering Bose gases in random potentials [LPZ04] (see also [Sto95, GHK05], GHK07], [SW16]), the study of non-interacting Bose gases (Bose gases without interaction between the particles) is nevertheless important since general features of BEC in the considered environment are revealed [LPZ04]. Generally, a main step in the proof of the occurrence of g-BEC in non-interacting Bose gases is to show that a certain critical density is finite. To determine the type of the condensate, however, is more difficult since this task requires a fairly accurate knowledge on how the eigenvalues of the corresponding one-particle Hamiltonian at the bottom of the spectrum behave in the thermodynamic limit.

In 1973 and 1974, Kac and Luttinger explored a non-interacting Bose gas in three dimensions in the presence of randomly distributed impurity centers which were assumed to represent hardcore potentials of finite range [KL73, KL74]. As a result, Kac and Luttinger were able to show that g-BEC occurs in probability for sufficiently low temperatures or, equivalently, for sufficiently large particle densities. Furthermore, they conjectured the existence of a macroscopic occupation of the ground state in this case but could not prove their assertion. They claimed that showing a certain lower bound for the gap between the two lowest eigenvalues of the respective one-particle random Schrdinger operator of the Bose gas would be sufficient to conclude a macroscopic occupation of the ground state. However, no further explanation was given to bolster this claim. Although it is indeed fairly easy to exclude a macroscopic occupation of excited states with the energy gap as assumed by Kac and Luttinger, it seems difficult to infer that a type-I BEC necessarily occurs. We suspect that Kac and Luttinger were not aware of the possibility that a type-III BEC [BL82, Ber83, BLP86, BLL86] can occur.

In this paper, we rigorously prove that a slightly stronger lower bound for the energy gap compared to the one assumed by Kac and Luttinger implies a macroscopic occupation of the ground state in probability and in the $r$ th mean. Consequently, confirming this lower bound for a given random model then proves the so-called Kac-Luttinger conjecture in that case. Our results are valid in all spatial dimensions $d \in \mathbb{N}$. We formulate our requirements for the random potential in a rather general framework. All of them are fulfilled, for example, by a Poisson random potential on $\mathbb{R}^{d}, d \in \mathbb{N}$, that has a non-negative, bounded, and compactly supported function as its single-impurity potential. The one-dimensional 
Luttinger-Sy [LS73a, LS73b] model (with infinite as well as finite interaction strength, see also [KPS19b]) also meets the requirement.

We formulate our general model and our assumptions for the random potential in Section 2. There, we also state and prove our main results, i.e., Theorem 2.7 and Corollary 2.9. In Section 3, we apply our results of Section 2 to the Luttinger-Sy model and show that in this model a type-I BEC, in which only the ground state is macroscopically occupied, occurs in probability and in the $r$ th mean. For the convenience of the reader we provide miscellaneous results that we use for proofs in this paper in Appendix A.

Finally, we remark that type-I BEC in the Luttinger-Sy model was previously investigated in [LZ07]. However, the authors used other methods and worked in a somewhat different setting resulting in a different thermodynamic limit.

\section{General model and results}

Let $(\Omega, \mathscr{A}, \mathbb{P})$ be a probability space. In this paper we shall consider self-adjoint random Schrdinger operators (acting as single-particle Hamiltonians) of the form

$$
H_{\omega}=-\Delta+V_{\omega}, \quad \omega \in \Omega,
$$

on $L^{2}\left(\mathbb{R}^{d}\right)$. The restriction of $H_{\omega}$ to a box $\Lambda_{N}:=\left(-L_{N}^{1 / d} / 2, L_{N}^{1 / d} / 2\right)^{d}, L_{N}>0$, with Dirichlet boundary conditions is given by the self-adjoint operator

$$
H_{N, \omega}=-\Delta+V_{\omega}, \quad \omega \in \Omega, \quad N \in \mathbb{N},
$$

on $L^{2}\left(\Lambda_{N}\right)$ with the Sobolev space $H_{0}^{1}\left(\Lambda_{N}\right)$ as its domain. Here, $-\Delta$ is the $d$-dimensional (Dirichlet-) Laplacian and $V_{\omega}$ is a random potential [FP92, Chapter 1]. We assume that $H_{N, \omega}$ is $\mathbb{P}$-almost surely a positive self-adjoint operator with purely discrete spectrum, i.e., with a spectrum that consists only of isolated eigenvalues of finite multiplicities. This is the case, for example, if $V_{\omega}$ is a Poisson random potential on $\mathbb{R}^{d}, d \in \mathbb{N}$, with a singleimpurity potential that is a bounded, compactly supported and non-negative function [LPZ04, Proposition 3.3], [FP92, Theorem 5.1]. These assumptions are also fulfilled if $V_{\omega}$ is, informally, a Poisson random potential on $\mathbb{R}$ with a singular single-impurity potential of the form $\gamma \delta$ where $\gamma>0$ or $\gamma=\infty$ denotes the interaction strength and $\delta$ the Dirac- $\delta$ distribution [FP92, p. 146], [LZ07].

We denote the associated sequence of eigenvalues of $H_{N, \omega}$, written in ascending order and each eigenvalue repeated according to its multiplicity, by $\left(E_{N}^{j, \omega}\right)_{j \in \mathbb{N}}$ and the associated sequence of eigenfunctions by $\left(\varphi_{N}^{j, \omega}\right)_{j \in \mathbb{N}}$. Note that $E_{N}^{j, \omega}>0$ and $\varphi_{N}^{j, \omega} \in L^{2}\left(\Lambda_{N}\right)$ for P-almost all $\omega \in \Omega$ and all $j, N \in \mathbb{N}$. Moreover, we indicate a random variable by writing an $\omega$ as a subscript or superscript. $\mathbb{E}$ refers to the expectation of a random variable with respect to P.

Remark 2.1. We also allow for Hamiltonians $H_{N, \omega}$ where the potential $V_{N, \omega}$ is distributionvalued as it is the case in the Luttinger-Sy model. In this case, one defines the Hamiltonian $H_{N, \omega}$ rigorously via its associated quadratic form on $H_{0}^{1}\left(\Lambda_{N}\right)$. 
We recall that the standard counting function is given by

$$
\mathcal{N}_{N}^{\mathrm{I}, \omega}(E):=\frac{1}{L_{N}}\left|\left\{j \in \mathbb{N}: E_{N}^{j, \omega}<E\right\}\right|, \quad \omega \in \Omega, \quad E \in \mathbb{R}
$$

where $|A|$ denotes the number of elements of a set $A \subset \mathbb{N}$. The function $\mathcal{N}_{N}^{\mathrm{I}, \omega}$ is called the integrated density of states and is $\mathbb{P}$-almost surely left-continuous and monotonically increasing. For $\mathbb{P}$-almost all $\omega \in \Omega, \mathcal{N}_{N}^{\mathrm{I}, \omega}$ consequently defines a unique Borel measure, that is, a measure on the Borel- $\sigma$-algebra on $\mathbb{R}$ such that the measure of any compact subset of $\mathbb{R}$ is finite. Ones denotes this uniquely defined measure by $\mathcal{N}_{N}^{\omega}$ and calls it the density of states.

Throughout this paper, we employ the standard thermodynamic limit: For $N \rightarrow \infty$ the volume $L_{N}$ of the box $\Lambda_{N}$ increases with the particle number $N$ of the system in such a way that the particle density $N / L_{N}=: \rho>0$ remains constant for all $N \in \mathbb{N}$.

In addition to what was mentioned above, we assume the following about $H_{N, \omega}$ in the rest of the paper:

\section{Assumptions 2.2.}

(i) For the ground-state energy $E_{N}^{1, \omega}$ of $H_{N}^{1, \omega}$, we have $\lim _{N \rightarrow \infty} E_{N}^{1, \omega}=0 \mathbb{P}$-almost surely.

(ii) There exists a non-random measure $\mathcal{N}_{\infty}$ such that $\mathrm{P}$-almost surely

$$
\lim _{N \rightarrow \infty} \mathcal{N}_{N}^{\omega}=\mathcal{N}_{\infty}
$$

in the vague sense; see Remark 2.3 .

(iii) There exist constants $c_{1}>0$ and $\widetilde{E}>0$ such that for all $N \in \mathbb{N}$ and all $0<E \leqslant \widetilde{E}$ one has

$$
\mathbb{E} \mathcal{N}_{N}^{\mathrm{I}, \omega}(E) \leqslant c_{1} \mathcal{N}_{\infty}^{\mathrm{I}}(E)
$$

Here,

$$
\mathcal{N}_{\infty}^{\mathrm{I}}(E):=\mathcal{N}_{\infty}((-\infty, E))= \begin{cases}\int_{(0, E)} \mathrm{d} \mathcal{N}_{\infty}(E) & \text { if } E>0 \\ 0 & \text { if } E \leqslant 0\end{cases}
$$

for all $E \in \mathbb{R}$.

(iv) There exists a constant $0<\eta_{1}<1$ such that

$$
\lim _{N \rightarrow \infty} N^{1-\eta_{1}} \mathcal{N}_{\infty}^{\mathrm{I}}\left(\left(\frac{\left(1+\eta_{1} / 2\right) \nu \gamma_{d}^{d / 2}}{\ln L_{N}}\right)^{2 / d}\right)=0,
$$

where $\gamma_{d}>0$ is the lowest Dirichlet eigenvalue of $-\Delta$ on the $d$-dimensional sphere with unit volume. 
We refer to Remark 2.12 for examples of random potentials for which these assumptions are met.

Remark 2.3. A sequence $\left(\mathscr{M}_{N}\right)_{N \in \mathbb{N}}$ of Radon measures (that is, of measures that are inner regular and locally finite) on the Borel- $\sigma$-algebra on $\mathbb{R}$ is said to converge to a Radon measure $\mathscr{M}_{\infty}$ in the vague sense if

$$
\lim _{N \rightarrow \infty} \int_{\mathbb{R}} f(E) \mathrm{d} \mathscr{M}_{N}(E)=\int_{\mathbb{R}} f(E) \mathrm{d} \mathscr{M}_{\infty}(E)
$$

for all continuous and compactly supported functions $f: \mathbb{R} \rightarrow \mathbb{R}$, see, e.g., [Bau01] for more details.

Remark 2.4. Assumption [2.2 (iv) implies in particular that

$$
\lim _{\epsilon \rightarrow 0^{+}} \epsilon^{-1} \mathcal{N}_{\infty}^{\mathrm{I}}(\epsilon)=0
$$

as well as

$$
\int_{0}^{\varepsilon} \mathcal{N}_{\infty}^{\mathrm{I}}(E) E^{-2} \mathrm{~d} E<\infty
$$

for arbitrary $\varepsilon>0$.

An important quantity in the context of BEC in non-interacting models is the so-called critical density $\rho_{c}(\beta)$ where $\beta:=1 / T \in(0, \infty)$ denotes the inverse temperature. With

$$
\mathcal{B}(E):=\left(\mathrm{e}^{\beta E}-1\right)^{-1} \mathbb{1}_{(0, \infty)}(E),
$$

$\mathbb{1}_{A}(E)$ being the indicator function of a measurable set $A \subset \mathbb{R}, \rho_{c}(\beta)$ is defined as

$$
\rho_{c}(\beta):=\int_{\mathbb{R}} \mathcal{B}(E) \mathrm{d} \mathcal{N}_{\infty}(E) .
$$

As usual, we study BEC in the grand-canonical ensemble of statistical mechanics. Hence, the number of particles occupying the $j$ th eigenstate is $\mathbb{P}$-almost surely given by

$$
n_{N}^{j, \omega}:=\left(\mathrm{e}^{\beta\left(E_{N}^{j, \omega}-\mu_{N}^{\omega}\right)}-1\right)^{-1}
$$

Here, $\mu_{N}^{\omega} \in\left(-\infty, E_{N}^{1, \omega}\right)$ is the so-called chemical potential which is, for $\mathrm{P}$-almost all $\omega \in \Omega$, uniquely determined by the condition that

$$
\frac{1}{L_{N}} \sum_{j=1}^{\infty} n_{N}^{j, \omega}=\rho
$$

holds for all values of $N \in \mathbb{N}$. We now state a first result which shows that the critical density as defined in (2.6) is indeed finite which consequently implies the existence of generalized BEC. For the proof we refer to [LPZ04, Theorem 4.1] in combination with [KPS19b, Theorem 2.7]. 
Theorem 2.5 (Generalized BEC). Under the above Assumptions 2.2 one has $\rho_{c}(\beta)<\infty$. In addition, if and only if the particle density is larger than the critical density, $\rho>\rho_{c}(\beta)$, generalized BEC $\mathrm{P}$-almost surely occurs, i.e., one has

$$
\mathbb{P}\left(\lim _{\epsilon \rightarrow 0^{+}} \liminf _{N \rightarrow \infty} \frac{1}{N} \sum_{j \in \mathbb{N}: E_{N}^{j, \omega}-E_{N}^{1, \omega} \leqslant \epsilon} n_{N}^{j, \omega}=\frac{\rho-\rho_{c}(\beta)}{\rho}>0\right)=1 .
$$

Moreover, whenever $\rho>\rho_{c}(\beta)$ the sequence of chemical potentials $\left(\mu_{N}^{\omega}\right)_{N \in \mathbb{N}} \mathbb{P}$-almost surely converges to zero.

In order to study the existence of macroscopic occupation of the ground state and to determine the type of condensation, we introduce the event

$$
\Omega_{N}^{c_{2}, c_{3}}:=\left\{\omega \in \Omega: E_{N}^{c_{2}+1, \omega}-E_{N}^{1, \omega} \geqslant c_{3} N^{-1+\eta_{1}} \text { and } E_{N}^{1, \omega} \leqslant\left[\left(1+\frac{\eta_{1}}{4}\right) \frac{\nu \gamma_{d}^{d / 2}}{\ln L_{N}}\right]^{2 / d}\right\}
$$

for some $c_{2} \in \mathbb{N}$ and $c_{3}>0$ and with the constant $\eta_{1}$ from Assumption 2.2 (iv). At this point we remark two facts: Firstly, the event $\Omega_{N}^{c_{2}, c_{3}}$ is the main ingredient in the "gap condition" as formulated in Theorem 2.7. Secondly, the upper bound for the ground-state energy $E_{N}^{1, \omega}$ in (2.10) is $\mathrm{P}$-almost surely fulfilled if $V_{\omega}$ is a Poisson random potential on $\mathbb{R}^{d}$ with a single-impurity potential that is a bounded, non-negative, compactly supported function, see for example [Szn98, Theorem 4.6].

Proposition 2.6. Under Assumptions 2.2, for $\rho>\rho_{c}(\beta)$, one has

$$
\liminf _{N \rightarrow \infty} \mathbb{E} \int_{\left(0, E_{N}^{c_{2}, \omega}\right]} \mathcal{B}\left(E-\mu_{N}^{\omega}\right) \mathrm{d} \mathcal{N}_{N}^{\omega}(E) \geqslant \rho-\rho_{c}(\beta)-\rho\left(1-\liminf _{N \rightarrow \infty} \mathbb{P}\left(\Omega_{N}^{c_{2}, c_{3}}\right)\right)
$$

Proof. For $\epsilon>0$ and $\eta_{1}$ from (2.10) we define the sets

$$
\begin{aligned}
& \widehat{\Omega}_{N}^{(1), \epsilon}:=\left\{\omega \in \Omega: E_{N}^{c_{2}, \omega} \geqslant \epsilon\right\}, \\
& \widehat{\Omega}_{N}^{(2), \epsilon}:=\left\{\omega \in \Omega: E_{N}^{c_{2}, \omega}<\epsilon\right\}, \\
& \widehat{\Omega}_{N}^{c_{2}, c_{3}}:=\widehat{\Omega}_{N}^{(2), \epsilon} \cap \Omega_{N}^{c_{2}, c_{3}} \cap\left\{\omega \in \Omega: E_{N}^{c_{2}+1, \omega} \geqslant\left[\left(1+\frac{\eta_{1}}{2}\right) \frac{\nu \gamma_{d}^{d / 2}}{\ln \left(L_{N}\right)}\right]^{2 / d}\right\},
\end{aligned}
$$

and

$$
\widetilde{\Omega}_{N}^{c_{2}, c_{3}}:=\widehat{\Omega}_{N}^{(2), \epsilon} \cap \Omega_{N}^{c_{2}, c_{3}} \cap\left\{\omega \in \Omega: E_{N}^{c_{2}+1, \omega}<\left[\left(1+\frac{\eta_{1}}{2}\right) \frac{\nu \gamma_{d}^{d / 2}}{\ln \left(L_{N}\right)}\right]^{2 / d}\right\}
$$


For all $\epsilon>0$ and all $N \in \mathbb{N}$ we conclude

$$
\begin{aligned}
\rho & =\int_{(0, \infty)} \mathcal{B}\left(E-\mu_{N}^{\omega}\right) \mathrm{d} \mathcal{N}_{N}^{\omega}(E) \\
& =\int_{(0, \epsilon]} \mathcal{B}\left(E-\mu_{N}^{\omega}\right) \mathrm{d} \mathcal{N}_{N}^{\omega}(E)+\int_{(\epsilon, \infty)} \mathcal{B}\left(E-\mu_{N}^{\omega}\right) \mathrm{d} \mathcal{N}_{N}^{\omega}(E)
\end{aligned}
$$

and thus

$$
\begin{aligned}
\rho \leqslant \int_{\hat{\Omega}_{N}^{(1), \epsilon}} & \int_{\left(0, E_{N}^{c_{2}, \omega}\right]} \mathcal{B}\left(E-\mu_{N}^{\omega}\right) \mathrm{d} \mathcal{N}_{N}^{\omega}(E) \mathrm{d} \mathbb{P}(\omega) \\
& +\int_{\hat{\Omega}_{N}^{(2), \epsilon}} \int_{\left(0, E_{N}^{c_{2}, \omega}\right]} \mathcal{B}\left(E-\mu_{N}^{\omega}\right) \mathrm{d} \mathcal{N}_{N}^{\omega}(E) \mathrm{d} \mathbb{P}(\omega) \\
& +\int_{\hat{\Omega}_{N}^{(2), \epsilon}} \int_{\left(E_{N}^{c_{2}, \omega}, \epsilon\right]} \mathcal{B}\left(E-\mu_{N}^{\omega}\right) \mathrm{d} \mathcal{N}_{N}^{\omega}(E) \mathrm{d} \mathbb{P}(\omega) \\
& +\mathbb{E} \int_{(\epsilon, \infty)} \mathcal{B}\left(E-\mu_{N}^{\omega}\right) \mathrm{d} \mathcal{N}_{N}^{\omega}(E) .
\end{aligned}
$$

Hence, we obtain

$$
\begin{aligned}
& \mathbb{E} \int_{\left(0, E_{N}^{c_{2}, \omega}\right]} \mathcal{B}\left(E-\mu_{N}^{\omega}\right) \mathrm{d} \mathcal{N}_{N}^{\omega}(E) \\
& \geqslant \rho- \int_{\hat{\Omega}_{N}^{(2), \epsilon} \cap \Omega_{N}^{c_{2}, c_{3}}} \int_{\left(E_{N}^{c_{2}, \omega}, \epsilon\right]} \mathcal{B}\left(E-\mu_{N}^{\omega}\right) \mathrm{d} \mathcal{N}_{N}^{\omega}(E) \mathrm{d} \mathbb{P}(\omega) \\
&-\int_{\hat{\Omega}_{N}^{(2), \epsilon} \cap \Omega \backslash \Omega_{N}^{c_{2}, c_{3}}} \int_{\left(E_{N}^{c_{2}, \omega}, \epsilon\right]} \mathcal{B}\left(E-\mu_{N}^{\omega}\right) \mathrm{d} \mathcal{N}_{N}^{\omega}(E) \mathrm{dP}(\omega) \\
&-\mathbb{E} \int_{(\epsilon, \infty)} \mathcal{B}\left(E-\mu_{N}^{\omega}\right) \mathrm{d} \mathcal{N}_{N}^{\omega}(E) \\
&=: \rho-A_{N}(\epsilon)-B_{N}(\epsilon)-C_{N}(\epsilon)
\end{aligned}
$$

for all $\epsilon>0$ and all $N \in \mathbb{N}$. In the following, we will discuss the $\operatorname{limit}_{\lim } \rightarrow 0+\lim \sup _{N \rightarrow \infty}$ of this equation.

Firstly, we state an upper bound for the term in line (2.15) in the considered limit. Let $\epsilon>0$ be arbitrarily given. For $\mathbb{P}$-almost all $\omega \in \Omega$ and all $N \in \mathbb{N}$, we have the bound 
$\int_{(\epsilon, \infty)} \mathcal{B}\left(E-\mu_{N}^{\omega}\right) \mathrm{d} \mathcal{N}_{N}^{\omega}(E) \leqslant \rho$. Employing Lemma A.1, we conclude that

$$
\limsup _{N \rightarrow \infty} \int_{(\epsilon, \infty)} \mathcal{B}\left(E-\mu_{N}^{\omega}\right) \mathrm{d} \mathcal{N}_{N}^{\omega}(E) \leqslant \int_{(\epsilon, \infty)} \mathcal{B}(E) \mathrm{d} \mathcal{N}_{\infty}(E)+\frac{2}{\beta \epsilon} \mathcal{N}_{\infty}^{\mathrm{I}}(\epsilon)
$$

P-almost surely. By using the (reverse) Fatou Lemma, we obtain

$$
\limsup _{N \rightarrow \infty} \mathbb{E} \int_{(\epsilon, \infty)} \mathcal{B}\left(E-\mu_{N}^{\omega}\right) \mathrm{d} \mathcal{N}_{N}^{\omega}(E) \leqslant \int_{(\epsilon, \infty)} \mathcal{B}(E) \mathrm{d} \mathcal{N}_{\infty}(E)+\frac{2}{\beta \epsilon} \mathcal{N}_{\infty}^{\mathrm{I}}(\epsilon) .
$$

Hence,

$$
\lim _{\epsilon \rightarrow 0+} \limsup _{N \rightarrow \infty} C_{N}(\epsilon) \leqslant \lim _{\epsilon \rightarrow 0+} \int_{(\epsilon, \infty)} \mathcal{B}(E) \mathrm{d} \mathcal{N}_{\infty}(E)=\int_{(0, \infty)} \mathcal{B}(E) \mathrm{d} \mathcal{N}_{\infty}(E)=\rho_{c}(\beta),
$$

where we used definitions (2.5) and (2.6) in the last step.

Next, we are concerned with the integral in line (2.13), and we are going to show that $\lim _{\epsilon \rightarrow 0+} \lim _{N \rightarrow \infty} A_{N}(\epsilon)=0$. Let $\epsilon>0$ be arbitrarily given. For convenience, we define

$$
\begin{aligned}
& A_{N}^{(1)}(\epsilon):=\int_{\widetilde{\Omega}_{N}^{c_{2}, c_{3}}} \int_{\left(E_{N}^{c_{2}, \omega},\left[\left(1+\eta_{1} / 2\right) \nu \gamma_{d}^{d / 2} / \ln \left(L_{N}\right)\right]^{2 / d}\right]} \mathcal{B}\left(E-\mu_{N}^{\omega}\right) \mathrm{d} \mathcal{N}_{N}^{\omega}(E) \mathrm{d} \mathbb{P}(\omega), \\
& A_{N}^{(2)}(\epsilon):=\int_{\widetilde{\Omega}_{N}^{c_{2}, c_{3}}} \int_{\left(\left[\left(1+\eta_{1} / 2\right) \nu \gamma_{d}^{d / 2} / \ln \left(L_{N}\right)\right]^{2 / d}, \epsilon\right]} \mathcal{B}\left(E-\mu_{N}^{\omega}\right) \mathrm{d} \mathcal{N}_{N}^{\omega}(E) \mathrm{dP}(\omega)
\end{aligned}
$$

for all $N \in \mathbb{N}$ large enough such that $\left[\left(1+\eta_{1} / 2\right) \nu \gamma_{d}^{d / 2} / \ln \left(L_{N}\right)\right]^{2 / d}<\epsilon$, and

$$
A_{N}^{(3)}(\epsilon):=\int_{\hat{\Omega}_{N}^{c_{2}, c_{3}}} \int_{\left(E_{N}^{c_{2}, \omega}, \epsilon\right]} \mathcal{B}\left(E-\mu_{N}^{\omega}\right) \mathrm{d} \mathcal{N}_{N}^{\omega}(E) \mathrm{d} \mathbb{P}(\omega) .
$$

Now, since $\mathcal{B}\left(E-\mu_{N}^{\omega}\right) \leqslant\left[\beta\left(E-E_{N}^{1, \omega}\right)\right]^{-1} \leqslant\left(\beta c_{3}\right)^{-1} N^{1-\eta_{1}}$ for all but finitely many $N \in \mathbb{N}$, all $\omega \in \Omega_{N}^{c_{2}, c_{3}}$, and all $E \geqslant E_{N}^{c_{2}+1, \omega}$, we obtain

$$
\begin{aligned}
\lim _{N \rightarrow \infty} A_{N}^{(1)}(\epsilon) & =\lim _{N \rightarrow \infty} \int_{\tilde{\Omega}_{N}^{c_{2}, c_{3}}} \int_{\left(E_{N}^{c_{2}, \omega},\left[\left(1+\eta_{1} / 2\right) \nu \gamma_{d}^{d / 2} / \ln \left(L_{N}\right)\right]^{2 / d}\right]} \mathcal{B}\left(E-\mu_{N}^{\omega}\right) \mathrm{d} \mathcal{N}_{N}^{\omega}(E) \mathrm{dP}(\omega) \\
& \leqslant\left(\beta c_{3}\right)^{-1} \lim _{N \rightarrow \infty} N^{1-\eta_{1}} \int_{\widetilde{\Omega}_{N}^{c_{2}, c_{3}}} \int_{\left.E_{N}^{c_{2}, \omega},\left[\left(1+\eta_{1} / 2\right) \nu \gamma_{d}^{d / 2} / \ln \left(L_{N}\right)\right]^{2 / d}\right]} \mathrm{d} \mathcal{N}_{N}^{\omega}(E) \mathrm{dP}(\omega) \\
& \leqslant\left(\beta c_{3}\right)^{-1} \lim _{N \rightarrow \infty} N^{1-\eta_{1}} \int_{\widetilde{\Omega}_{N}^{c_{2}, c_{3}}} \mathcal{N}_{N}^{\mathrm{I}, \omega}\left(\left(\frac{\left(1+\eta_{1} / 2\right) \nu \gamma_{d}^{d / 2}}{\ln \left(L_{N}\right)}\right)^{2 / d}\right) \mathrm{d} \mathbb{P}(\omega) .
\end{aligned}
$$


Using the fact that $\mathcal{N}_{N}^{\mathrm{I}, \omega}(E) \geqslant 0$ for $\mathbb{P}$-almost all $\omega \in \Omega$, for all $E \in \mathbb{R}$, and for all $N \in \mathbb{N}$ as well as employing our conditions (iii) and (iv) from Assumptions 2.2, we get

$$
\begin{aligned}
\lim _{N \rightarrow \infty} A_{N}^{(1)}(\epsilon) & \leqslant\left(\beta c_{3}\right)^{-1} \lim _{N \rightarrow \infty} N^{1-\eta_{1}} \mathbb{E} \mathcal{N}_{N}^{\mathrm{I}, \omega}\left(\left(\frac{\left(1+\eta_{1} / 2\right) \nu \gamma_{d}^{d / 2}}{\ln \left(L_{N}\right)}\right)^{2 / d}\right) \\
& \leqslant\left(\beta c_{3}\right)^{-1} c_{1} \lim _{N \rightarrow \infty} N^{1-\eta_{1}} \mathcal{N}_{\infty}^{\mathrm{I}}\left(\left(\frac{\left(1+\eta_{1} / 2\right) \nu \gamma_{d}^{d / 2}}{\ln \left(L_{N}\right)}\right)^{2 / d}\right) \\
& =0 .
\end{aligned}
$$

Secondly, for arbitrary $N \in \mathbb{N}$ we conclude that for all $E \geqslant\left[\left(1+\eta_{1} / 2\right) \nu \gamma_{d}^{d / 2} / \ln \left(L_{N}\right)\right]^{2 / d}$ and for all $\omega \in \widetilde{\Omega}_{N}^{c_{2}, c_{3}}$,

$$
E \geqslant\left(\left(1+\frac{\eta_{1}}{2}\right) \frac{\nu \gamma_{d}^{d / 2}}{\ln \left(L_{N}\right)}\right)^{2 / d} \geqslant\left(\frac{1+\eta_{1} / 2}{1+\eta_{1} / 4}\right)^{2 / d} E_{N}^{1, \omega}
$$

and, consequently,

$$
E-\mu_{N}^{\omega} \geqslant E-E_{N}^{1, \omega} \geqslant\left[1-\left(\frac{1+\eta_{1} / 4}{1+\eta_{1} / 2}\right)^{2 / d}\right] E=c_{4} E,
$$

where we have set $c_{4}:=1-\left(\left[1+\eta_{1} / 4\right] /\left[1+\eta_{1} / 2\right]\right)^{2 / d}>0$. Hence,

$$
\begin{aligned}
& \lim _{\epsilon \rightarrow 0+} \limsup _{N \rightarrow \infty} A_{N}^{(2)}(\epsilon) \\
& =\lim _{\epsilon \rightarrow 0+} \limsup _{N \rightarrow \infty} \int_{\widetilde{\Omega}_{N}^{c_{2}, c_{3}}} \int_{\left(\left[\left(1+\eta_{1} / 2\right) \nu \gamma_{d}^{d / 2} / \ln \left(L_{N}\right)\right]^{2 / d}, \epsilon\right]} \mathcal{B}\left(E-\mu_{N}^{\omega}\right) \mathrm{d} \mathcal{N}_{N}^{\omega}(E) \mathrm{d} \mathbb{P}(\omega) \\
& \leqslant \lim _{\epsilon \rightarrow 0+} \limsup _{N \rightarrow \infty} \int_{\widetilde{\Omega}_{N}^{c_{2}, c_{3}}} \int_{\left(\left[\left(1+\eta_{1} / 2\right) \nu \gamma_{d}^{d / 2} / \ln \left(L_{N}\right)\right]^{2 / d}, \epsilon\right]} \mathcal{B}\left(c_{4} E\right) \mathrm{d} \mathcal{N}_{N}^{\omega}(E) \mathrm{dP}(\omega) \\
& \leqslant\left(\beta c_{4}\right)^{-1} \lim _{\epsilon \rightarrow 0+} \limsup _{N \rightarrow \infty} \int_{\widetilde{\Omega}_{N}^{c_{2}, c_{3}}} \int_{\left(\left[\left(1+\eta_{1} / 2\right) \nu \gamma_{d}^{d / 2} / \ln \left(L_{N}\right)\right]^{2 / d}, \epsilon\right]} E^{-1} \mathrm{~d} \mathcal{N}_{N}^{\omega}(E) \mathrm{dP}(\omega) \text {. }
\end{aligned}
$$

Employing an integration by parts (for Lebesgue-Stieltjes integrals, see, e.g., [HS65, The- 
orem 21.67]), we have

$$
\begin{aligned}
\lim _{\epsilon \rightarrow 0+} \limsup _{N \rightarrow \infty} A_{N}^{(2)}(\epsilon) & \\
\leqslant\left(\beta c_{4}\right)^{-1} \lim _{\epsilon \rightarrow 0+} \limsup _{N \rightarrow \infty} \int_{\Omega_{N}^{c_{2}, c_{3}}} & {\left[\epsilon^{-1} \mathcal{N}_{N}^{\mathrm{I}, \omega}(2 \epsilon)\right.} \\
& \left.+\int_{\left[\left(1+\eta_{1} / 2\right) \nu \gamma_{d}^{d / 2} / \ln \left(L_{N}\right)\right]^{2 / d}}^{\epsilon} \mathcal{N}_{N}^{\mathrm{I}, \omega}(E) E^{-2} \mathrm{~d} E\right] \mathrm{dP}(\omega) .
\end{aligned}
$$

We then use again the fact that $\mathcal{N}_{N}^{\mathrm{I}, \omega}(E) \geqslant 0$ for $\mathbb{P}$-almost all $\omega \in \Omega$, all $E \in \mathbb{R}$, and all $N \in \mathbb{N}$ and employ Assumption 2.2 (iii) and Remark 2.4 to obtain

$$
\begin{aligned}
& \lim _{\epsilon \rightarrow 0+} \limsup _{N \rightarrow \infty} A_{N}^{(2)}(\epsilon) \\
& \leqslant\left(\beta c_{4}\right)^{-1} \lim _{\epsilon \rightarrow 0+} \limsup _{N \rightarrow \infty}\left[\epsilon^{-1} \int_{\widetilde{\Omega}_{N}^{c_{2}, c_{3}}} \mathcal{N}_{N}^{\mathrm{I}, \omega}(2 \epsilon) \mathrm{dP}(\omega)\right. \\
& \left.+\int_{\left[\left(1+\eta_{1} / 2\right) \nu \gamma_{d}^{d / 2} / \ln \left(L_{N}\right)\right]^{2 / d}}^{\epsilon} E^{-2} \int_{\widetilde{\Omega}_{N}^{c_{2}, c_{3}}} \mathcal{N}_{N}^{\mathrm{I}, \omega}(E) \mathrm{dP}(\omega) \mathrm{d} E\right] \\
& \leqslant\left(\beta c_{4}\right)^{-1} \lim _{\epsilon \rightarrow 0+} \limsup _{N \rightarrow \infty}\left[\epsilon^{-1} \mathbb{E} \mathcal{N}_{N}^{\mathrm{I}, \omega}(2 \epsilon)\right. \\
& \left.+\int_{\left[\left(1+\eta_{1} / 2\right) \nu \gamma_{d}^{d / 2} / \ln \left(L_{N}\right)\right]^{2 / d}}^{\epsilon} E^{-2} \mathbb{E} \mathcal{N}_{N}^{\mathrm{I}, \omega}(E) \mathrm{d} E\right] \\
& \leqslant \frac{c_{1}}{\beta c_{4}} \lim _{\epsilon \rightarrow 0+}\left[\epsilon^{-1} \mathcal{N}_{\infty}^{\mathrm{I}}(2 \epsilon)+\int_{0}^{\epsilon} \mathcal{N}_{\infty}^{\mathrm{I}}(E) E^{-2} \mathrm{~d} E\right] \\
& =0 \text {. }
\end{aligned}
$$

In a third step we show that

$$
\lim _{\epsilon \rightarrow 0+} \limsup _{N \rightarrow \infty} A_{N}^{(3)}(\epsilon)=0
$$

proceeding similarly as before. Note that for all $N \in \mathbb{N}$, all $\omega \in \widehat{\Omega}_{N}^{c_{2}, c_{3}}$, and all $E>E_{N}^{c_{2}+1, \omega}$ we now have

$$
E \geqslant E_{N}^{c_{2}+1, \omega} \geqslant\left(\left(1+\frac{\eta_{1}}{2}\right) \frac{\nu \gamma_{d}^{d / 2}}{\ln \left(L_{N}\right)}\right)^{2 / d} \geqslant\left(\frac{1+\eta_{1} / 2}{1+\eta_{1} / 4}\right)^{2 / d} E_{N}^{1, \omega}
$$


and hence

$$
E-\mu_{N}^{\omega} \geqslant E-E_{N}^{1, \omega} \geqslant\left[1-\left(\frac{1+\eta_{1} / 4}{1+\eta_{1} / 2}\right)^{2 / d}\right] E=c_{4} E
$$

Consequently,

$$
\lim _{\epsilon \rightarrow 0+} \limsup _{N \rightarrow \infty} A_{N}^{(3)}(\epsilon) \leqslant \frac{c_{1}}{\beta c_{4}} \lim _{\epsilon \rightarrow 0+}\left[\epsilon^{-1} \mathcal{N}_{\infty}^{\mathrm{I}}(2 \epsilon)+\int_{0}^{\epsilon} \mathcal{N}_{\infty}^{\mathrm{I}}(E) E^{-2} \mathrm{~d} E\right]=0 .
$$

Summing up the last steps we have shown that $\lim _{\epsilon \rightarrow 0+} \lim \sup _{N \rightarrow \infty} A_{N}(\epsilon)=0$.

In a final step we bound the term in line (2.14): For all $\epsilon>0$,

$$
\begin{aligned}
\limsup _{N \rightarrow \infty} B_{N}(\epsilon) & \leqslant \limsup _{N \rightarrow \infty} \int_{\hat{\Omega}_{N}^{(2), \epsilon} \cap \Omega \backslash \Omega_{N}^{c_{2}, c_{3}}} \int_{(0, \epsilon]} \mathcal{B}\left(E-\mu_{N}^{\omega}\right) \mathrm{d} \mathcal{N}_{N}^{\omega}(E) \mathrm{d} \mathbb{P}(\omega) \\
& \leqslant \rho \limsup _{N \rightarrow \infty} \mathbb{P}\left(\Omega \backslash \Omega_{N}^{c_{2}, c_{3}}\right) .
\end{aligned}
$$

Thus,

$$
\liminf _{N \rightarrow \infty} \mathbb{E} \int_{\left(0, E_{N}^{c_{2}, \omega}\right]} \mathcal{B}\left(E-\mu_{N}^{\omega}\right) \mathrm{d} \mathcal{N}_{N}^{\omega}(E) \geqslant \rho-\rho_{c}-\rho \limsup _{N \rightarrow \infty} \mathbb{P}\left(\Omega \backslash \Omega_{N}^{c_{2}, c_{3}}\right)
$$

We can now state our main result which allows to conclude that, for particle densities larger than the critical one and given a certain "gap condition" is satisfied, one indeed has BEC of type I in probability.

Theorem 2.7 (Type-I BEC in probability). Assume that Assumptions 2.2 are fulfilled and that $\rho>\rho_{c}(\beta)$. If $H_{N, \omega}$ is such that the "gap condition" for some $c_{2} \in \mathbb{N}$ and $c_{3}>0$ is fulfilled, i.e.,

$$
\lim _{N \rightarrow \infty} \mathbb{P}\left(\Omega_{N}^{c_{2}, c_{3}}\right)=1,
$$

then, for all $\eta>0$,

$$
\lim _{N \rightarrow \infty} \mathbb{P}\left(\left|\frac{1}{N} \sum_{j=1}^{c_{2}} n_{N}^{j, \omega}-\frac{\rho-\rho_{c}(\beta)}{\rho}\right|<\eta\right)=1
$$

In addition, for all $\eta>0$ and all $j \geqslant c_{2}+1$ one has

$$
\lim _{N \rightarrow \infty} \mathbb{P}\left(\frac{n^{j, \omega}}{N} \geqslant \eta\right)=0
$$

In other words, one has type-I BEC in probability if the gap condition (2.17) is fulfilled. 
Proof. We define $\rho_{0}(\beta):=\rho-\rho_{c}(\beta)$ for convenience. Assumption (2.17) together with Proposition 2.6 imply that

$$
\liminf _{N \rightarrow \infty} \mathbb{E} \int_{\left(0, E_{N}^{c_{2}, \omega}\right]} \mathcal{B}\left(E-\mu_{N}^{\omega}\right) \mathrm{d} \mathcal{N}_{N}^{\omega}(E) \geqslant \rho_{0}(\beta)
$$

On the other hand, with Lemma A.2, the (reverse) Fatou Lemma, and the fact that

$$
\int_{\left(0, E_{N}^{c_{2}, \omega}\right]} \mathcal{B}\left(E-\mu_{N}^{\omega}\right) \mathrm{d} \mathcal{N}_{N}^{\omega}(E) \leqslant \rho
$$

for P-almost all $\omega \in \Omega$ and for all $N \in \mathbb{N}$, we obtain

$$
\begin{aligned}
\limsup _{N \rightarrow \infty} \mathbb{E} \int_{\left(0, E_{N}^{c_{2}, \omega}\right]} \mathcal{B}\left(E-\mu_{N}^{\omega}\right) \mathrm{d} \mathcal{N}_{N}^{\omega}(E) & \leqslant \mathbb{E} \limsup _{N \rightarrow \infty} \int_{\left(0, E_{N}^{c_{2}, \omega}\right]} \mathcal{B}\left(E-\mu_{N}^{\omega}\right) \mathrm{d} \mathcal{N}_{N}^{\omega}(E) \\
& \leqslant \rho_{0}(\beta) .
\end{aligned}
$$

In conclusion, remembering the relation $\rho=N / L_{N}$,

$$
\lim _{N \rightarrow \infty} \mathbb{E} \int_{\left(0, E_{N}^{c_{2}, \omega}\right]} \mathcal{B}\left(E-\mu_{N}^{\omega}\right) \mathrm{d} \mathcal{N}_{N}^{\omega}(E)=\lim _{N \rightarrow \infty} \mathbb{E} \frac{\rho}{N} \sum_{j=1}^{c_{2}} n_{N}^{j, \omega}=\rho_{0}(\beta)
$$

In addition, for an arbitrary $\tilde{\eta}>0$ and with Lemma A.2 we obtain

$$
\begin{aligned}
& 0 \leqslant \limsup _{N \rightarrow \infty} \int_{\frac{1}{N} \sum_{j=1}^{c_{2}} n_{N}^{j, \omega}>\rho_{0}(\beta) / \rho}\left(\frac{1}{N} \sum_{j=1}^{c_{2}} n_{N}^{j, \omega}-\frac{\rho_{0}(\beta)}{\rho}\right) \mathrm{d} \mathbb{P}(\omega) \\
& \leqslant \limsup _{N \rightarrow \infty} \int_{\rho_{0}(\beta) / \rho<\frac{1}{N} \sum_{j=1}^{c_{2}}}\left(\frac{1}{N} \sum_{j=1}^{c_{2}} n_{N}^{j, \omega}-\frac{\rho_{0}(\beta)}{\rho}\right) \mathrm{d} \mathbb{P}(\omega) \\
& \quad+\limsup _{N \rightarrow \infty} \int_{\left.\frac{1}{N} \sum_{j=1}^{c_{2}} n_{n_{N}}^{j, \omega} \geqslant\left(\rho_{0}(\beta) / \rho\right)+\tilde{\eta}\right)+\widetilde{\eta}}\left(\frac{1}{N} \sum_{j=1}^{c_{2}} n_{N}^{j, \omega}-\frac{\rho_{0}(\beta)}{\rho}\right) \mathrm{d} \mathbb{P}(\omega) \\
& \leqslant \widetilde{\eta}+\left(1-\frac{\rho_{0}(\beta)}{\rho}\right) \lim _{N \rightarrow \infty} \mathbb{P}\left(\frac{1}{N} \sum_{j=1}^{c_{2}} n_{N}^{j, \omega} \geqslant \frac{\rho_{0}(\beta)}{\rho}+\widetilde{\eta}\right) \\
& \leqslant \widetilde{\eta} .
\end{aligned}
$$


Thus, by (2.21),

$$
\begin{aligned}
0 & =\lim _{N \rightarrow \infty} \mathbb{E}\left[\frac{1}{N} \sum_{j=1}^{c_{2}} n_{N}^{j, \omega}-\frac{\rho_{0}(\beta)}{\rho}\right]=\lim _{N \rightarrow \infty} \int_{\Omega}\left(\frac{1}{N} \sum_{j=1}^{c_{2}} n_{N}^{j, \omega}-\frac{\rho_{0}(\beta)}{\rho}\right) \mathrm{d} \mathbb{P}(\omega) \\
& =\lim _{N \rightarrow \infty} \int_{\frac{1}{N} \sum_{j=1}^{c_{2}} n_{N}^{j, \omega} \leqslant \rho_{0}(\beta) / \rho}\left(\frac{1}{N} \sum_{j=1}^{c_{2}} n_{N}^{j, \omega}-\frac{\rho_{0}(\beta)}{\rho}\right) \mathrm{dP}(\omega) .
\end{aligned}
$$

We conclude

$$
\lim _{N \rightarrow \infty} \mathbb{E}\left|\frac{1}{N} \sum_{j=1}^{c_{2}} n_{N}^{j, \omega}-\frac{\rho_{0}(\beta)}{\rho}\right|=0
$$

and, consequently, for all $\eta>0$,

$$
\lim _{N \rightarrow \infty} \mathbb{P}\left(\left|\frac{1}{N} \sum_{j=1}^{c_{2}} n_{N}^{j, \omega}-\frac{\rho_{0}(\beta)}{\rho}\right|<\eta\right)=1 .
$$

Finally, we show the last part of the theorem: Using the fact that $N^{-1} \sum_{j=1}^{c_{2}+1} n_{N}^{j, \omega} \leqslant 1$ for P-almost all $\omega \in \Omega$ and for all $N \in \mathbb{N}$, Lemma A.2, and the (reverse) Fatou Lemma we obtain

$$
\limsup _{N \rightarrow \infty} \mathbb{E} \frac{1}{N} \sum_{j=1}^{c_{2}+1} n_{N}^{j, \omega} \leqslant \frac{\rho_{0}(\beta)}{\rho}
$$

In addition,

$$
\liminf _{N \rightarrow \infty} \mathbb{E} \frac{1}{N} \sum_{j=1}^{c_{2}+1} n_{N}^{j, \omega} \geqslant \lim _{N \rightarrow \infty} \mathbb{E} \frac{1}{N} \sum_{j=1}^{c_{2}} n_{N}^{j, \omega}=\frac{\rho_{0}(\beta)}{\rho},
$$

see (2.21). Therefore,

$$
\frac{\rho_{0}(\beta)}{\rho}=\lim _{N \rightarrow \infty} \mathbb{E} \frac{1}{N} \sum_{j=1}^{c_{2}+1} n_{N}^{j, \omega}=\frac{\rho_{0}(\beta)}{\rho}+\lim _{N \rightarrow \infty} \mathbb{E} \frac{n_{N}^{c_{2}+1, \omega}}{N} .
$$

For any $j \geqslant c_{2}+1$ we thus conclude, due to the fact that $n_{N}^{j, \omega} \leqslant n_{N}^{c_{2}+1, \omega}$ for $\mathbb{P}$-almost all $\omega \in \Omega$ and for all $N \in \mathbb{N}$,

$$
\lim _{N \rightarrow \infty} \mathbb{E}\left|\frac{n_{N}^{j, \omega}}{N}\right|=0
$$

and, in particular, for all $\eta>0$,

$$
\lim _{N \rightarrow \infty} \mathbb{P}\left(\frac{n_{N}^{j, \omega}}{N}<\eta\right)=1
$$


Remark 2.8. Since $c_{2} n_{N}^{1, \omega} \geqslant \sum_{j=1}^{c_{2}} n_{N}^{j, \omega}$ for $\mathbb{P}$-almost all $\omega \in \Omega$ and for all $N \in \mathbb{N}$, Theorem 2.7 immediately implies that, for $\rho>\rho_{c}$ and for all $\eta>0$,

$$
\lim _{N \rightarrow \infty} \mathbb{P}\left(\frac{1}{N} n_{N}^{1, \omega}>c_{2}^{-1} \frac{\rho-\rho_{c}(\beta)}{\rho}-\eta\right)=1 .
$$

This shows that the ground state is macroscopically occupied in probability.

In a next result we establish another consequence of Theorem 2.7, namely, existence of $\mathrm{BEC}$ in the $r t h$ mean.

Corollary 2.9 (Type-I BEC in the $r$ th mean). Assume that $\rho>\rho_{c}(\beta)$ and that the gap condition (2.17) is fulfilled. Then, for all $r \geqslant 1$, one has

$$
\lim _{N \rightarrow \infty} \mathbb{E}\left|\frac{1}{N} \sum_{j=1}^{c_{2}} n_{N}^{j, \omega}-\frac{\rho-\rho_{c}(\beta)}{\rho}\right|^{r}=0
$$

as well as

$$
\lim _{N \rightarrow \infty} \mathbb{E}\left|\frac{n_{N}^{j, \omega}}{N}\right|^{r}=0
$$

for every $j \geqslant c_{2}$.

Proof. The proof readily follows from Theorem 2.7 taking into account standard results from probability theory. Namely, from Theorem 2.7 we conclude that the two sequences of random variables

$$
\left(\frac{1}{N} \sum_{j=1}^{c_{2}} n_{N}^{j, \omega}-\frac{\rho-\rho_{c}(\beta)}{\rho}\right)_{N \in \mathbb{N}} \text { and }\left(\frac{n_{N}^{c_{2}+1, \omega}}{N}\right)_{N \in \mathbb{N}}
$$

converge to zero in probability. In addition, since

$$
-1 \leqslant \frac{1}{N} \sum_{j=1}^{c_{2}} n_{N}^{j, \omega}-\frac{\rho-\rho_{c}(\beta)}{\rho} \leqslant 1
$$

and

$$
0 \leqslant \frac{n_{N}^{c_{2}+1, \omega}}{N} \leqslant 1
$$

for $\mathbb{P}$-almost all $\omega \in \Omega$, both sequences are $\mathbb{P}$-almost surely uniformly bounded which then implies the statement.

Corollary 2.10 (Almost sure macroscopic occupation of the ground state). If the requirements of Theorem 2.7 are fulfilled, then the ground state is $\mathbb{P}$-almost surely macroscopically occupied,

$$
\mathbb{P}\left(\limsup _{N \rightarrow \infty} \frac{n_{N}^{1, \omega}}{N}>0\right)=1
$$


Proof. The statement can be proved in a similar vein as [KPS19b, Theorem 3.5], taking Remark 2.8 into account.

Alternatively, (2.18) implies, for P-almost all $\omega \in \Omega$, the existence of a subsequence $\left(\frac{1}{N_{k}} \sum_{j=1}^{c_{2}} n_{N_{k}}^{j, \omega}\right)_{k \in \mathbb{N}}$ converging to $\frac{\rho-\rho_{c}(\beta)}{\rho}$ in the limit $k \rightarrow \infty$. Consequently,

$$
\frac{\rho-\rho_{c}(\beta)}{\rho} \leqslant \limsup _{N \rightarrow \infty} \frac{1}{N} \sum_{j=1}^{c_{2}} n_{N}^{j, \omega} \leqslant c_{2} \limsup _{N \rightarrow \infty} \frac{n_{N}^{1, \omega}}{N}
$$

P-almost surely, which implies the statement.

Remark 2.11. By the reverse triangle inequality we also see the following: Given the requirements in Theorem 2.7 are met, then

$$
\lim _{N \rightarrow \infty} \mathbb{E}\left(\frac{1}{N} \sum_{j=1}^{c_{2}} n_{N}^{j, \omega}\right)^{r}=\mathbb{E}\left(\frac{\rho-\rho_{c}(\beta)}{\rho}\right)^{r}
$$

for all $r \geqslant 1$. In particular,

$$
\liminf _{N \rightarrow \infty} \mathbb{E} \frac{n_{N}^{1, \omega}}{N} \geqslant \frac{1}{c_{2}} \frac{\rho-\rho_{c}(\beta)}{\rho},
$$

i.e., the ground state is macroscopically occupied in expectation.

Remark 2.12. A Poisson random potential on $\mathbb{R}^{d}, d \in \mathbb{N}$, with a single-impurity potential that is a non-negative, compactly supported, and bounded function fulfills all requirements of Assumptions [2.2, see, for example, [Szn98, Theorem 4.6], [FP92, Theorem 5.20, 5.25, and Theorem 10.2], as well as [LMW03]. Theorem 2.7 and Corollaries 2.9 and 2.10 thus apply to such a Poisson random potential. To the best of our knowledge, however, it is so far not known whether the "gap condition" of Theorem 2.7 is then valid. In the next chapter, we study a special case of Poisson random potentials on $\mathbb{R}$ for which we are able to confirm the "gap condition."

\section{An example: the Luttinger-Sy model}

In this section we are concerned with BEC in the Luttinger-Sy model (LS-model) LS73a, LS73b for which all the assumptions on the Hamiltonian $H_{N, \omega}$, see Assumptions 2.2 and before, are fulfilled. It is our aim to show that also the "gap condition" as formulated in Theorem 2.7 is realized in the LS-model. Hence, the corresponding versions of Theorem 2.7 and Corollaries 2.9 and 2.10 hold for the LS-model, too.

The Luttinger-Sy model is a random one-dimensional model which is obtained by dissecting the real line $\mathbb{R}$ into a ( $\mathbb{P}$-almost surely) countable number of intervals via a set of points $\left\{\hat{x}_{j}(\omega)\right\}_{j}$ generated by a Poisson point process of intensity $\nu>0$. These points can $\mathbb{P}$-almost surely be labeled by $\mathbb{Z}$ such that

$$
\ldots<\hat{x}_{-1}(\omega)<\hat{x}_{0}(\omega)<0<\hat{x}_{1}(\omega)<\hat{x}_{2}(\omega)<\ldots .
$$


We refer to [Kin93, Chapter 4] for more details regarding the Poisson point process on $\mathbb{R}$. At each point $\hat{x}_{j}(\omega)$ one then imposes a Dirichlet boundary condition which is informally equivalent to saying that one places a Dirac- $\delta$ potential of infinite strength $\gamma=\infty$ at each Poisson point $\hat{x}_{j}(\omega)$. Hence, the one-particle Hamiltonian in the LS-model is informally given by

$$
H_{\omega}=-\frac{\mathrm{d}^{2}}{\mathrm{~d} x^{2}}+\gamma \sum_{j \in \mathbb{Z}} \delta\left(x-\hat{x}_{j}(\omega)\right), \quad \omega \in \Omega, \quad \gamma=\infty,
$$

$(\Omega, \mathscr{A}, \mathbb{P})$ denoting the underlying probability space. BEC is investigated employing a thermodynamic limit which makes it necessary - in a first step - to restrict $H_{\omega}$ to finite volume. For this one introduces the window $\Lambda_{N}:=\left(-L_{N} / 2,+L_{N} / 2\right) \subset \mathbb{R}$ where the length $L_{N}$ is determined through the relation $L_{N}:=N / \rho, N \in \mathbb{N}$ denotes the number of particles, and $\rho>0$ is the particle density. On $L^{2}\left(\Lambda_{N}\right)$ one then introduces the (informal) finite-volume one-particle Hamiltonian

$$
H_{N, \omega}:=-\frac{\mathrm{d}^{2}}{\mathrm{~d} x^{2}}+\gamma \sum_{j \in \mathbb{Z}: \hat{x}_{j}(\omega) \in \Lambda_{N}} \delta\left(x-\hat{x}_{j}(\omega)\right), \quad \omega \in \Omega, \quad \gamma=\infty .
$$

We refer to [LZ07, KPS19a] for a rigorous realization of $H_{N, \omega}$ as a self-adjoint operator.

Since one imposes Dirichlet boundary conditions at each point $\hat{x}_{j}(\omega)$, the window $\Lambda_{N}$ effectively consists of a collection of smaller intervals of lengths $l_{N}^{j, \omega}:=\mid\left(\hat{x}_{j}(\omega), \hat{x}_{j+1}(\omega)\right) \cap$ $\Lambda_{N}$ | on each of which the operator $H_{N, \omega}$ acts as the standard one-dimensional Dirichlet Laplacian. As a consequence, the eigenvalues of $H_{N, \omega}$ are just the collection of all the eigenvalues coming from these Dirichlet Laplacians; their eigenvalues, on the other hand, are explicitly given: namely, the $n$th eigenvalue on the interval with length $l_{N}^{j, \omega}$ simply is $\left(\pi n / l_{N}^{j, \omega}\right)^{2}, n \in \mathbb{N}$

We denote the intervals generated by the Poisson point process within $\Lambda_{N}$ by

$$
I_{N}^{j, \omega}:=\left(\hat{x}_{j}(\omega), \hat{x}_{j+1}(\omega)\right) \cap \Lambda_{N}
$$

for all $j \in \mathbb{Z}$ and all $N \in \mathbb{N}$. Also, let $l_{N,>}^{k, \omega}, k \in \mathbb{N}$, be the $k$ th largest length of these intervals. We denote by $\kappa_{N}^{\omega}$ the number of Poisson points within $\Lambda_{N}$. Consequently, for P-almost all $\omega \in \Omega$ and for all $N \in \mathbb{N}$ there are $\kappa_{N}^{\omega}+1$ many lengths $l_{N}^{j, \omega}$ that are larger than zero. Furthermore, $\left\{\hat{l}^{j, \omega}: j \in \mathbb{Z} \backslash\{0\}\right\} \cup\left\{\left|\left(\hat{x}_{0}(\omega), 0\right)\right|\right\} \cup\left\{\left|\left(0, \hat{x}_{1}(\omega)\right)\right|\right\}$ where $\hat{l}^{j, \omega}:=$ $\mid\left(\hat{x}_{j}(\omega), \hat{x}_{j+1}(\omega) \mid\right.$ are mutually independent, exponentially distributed random variables with common probability density $\nu \mathrm{e}^{-\nu l}$ [Kin93, Section 4.1].

Another main advantage of considering the LS-model is that there is an explicit expression for the integrated density of states: One has

$$
\mathcal{N}_{\infty}^{\mathrm{I}}(E)=\nu \frac{\mathrm{e}^{-\nu \pi E^{-1 / 2}}}{1-\mathrm{e}^{-\nu \pi E^{-1 / 2}}} \mathbb{1}_{(0, \infty)}(E), \quad E \in \mathbb{R},
$$

see [LPZ04, Proposition 3.2] and also [Egg72, LS73a, LS73b.

Now we state a version of Theorem 2.7 for the LS-model. For this, recall the "gap condition" (2.10). 
Theorem 3.1 (Gap condition in the LS-model). In the LS-model one has

$$
\lim _{N \rightarrow \infty} \mathbb{P}\left(\Omega_{N}^{1,1}\right)=1
$$

Proof. We will prove that the gap condition is actually fulfilled for any value $0<\eta_{1}<1 / 2$ of the constant $\eta_{1}$ appearing in Assumptions 2.2 (iv) and definition (2.10).

Let $0<\eta_{1}<1 / 2$ be arbitrary. We conclude with Lemma A.5 that

$$
\mathbb{P}\left(\lim _{N \rightarrow \infty}\left[\left(1+\frac{\eta_{1}}{3}\right)^{-1} \frac{\ln \left(L_{N}\right)}{\nu}-l_{N,>}^{1, \omega}\right]_{+}=0\right)=1
$$

and consequently, for an arbitrary $\eta>0$,

$$
\begin{aligned}
\lim _{N \rightarrow \infty} \mathbb{P}\left(l_{N,>}^{1, \omega} \leqslant\left(1+\frac{\eta_{1}}{2}\right)^{-1} \frac{\ln \left(L_{N}\right)}{\nu}\right) & \leqslant \lim _{N \rightarrow \infty} \mathbb{P}\left(\left[\left(1+\frac{\eta_{1}}{3}\right)^{-1} \frac{\ln \left(L_{N}\right)}{\nu}-l_{N,>}^{1, \omega}\right]_{+}>\eta\right) \\
& =0
\end{aligned}
$$

Therefore, since $E_{N}^{1, \omega}=\pi^{2} /\left(l_{N,>}^{1, \omega}\right)^{2}$,

$$
\lim _{N \rightarrow \infty} \mathbb{P}\left(E_{N}^{1, \omega} \leqslant\left[\left(1+\frac{\eta_{1}}{2}\right) \frac{\nu \pi}{\ln \left(L_{N}\right)}\right]^{2}\right)=1 .
$$

The show the second part of the gap condition, we introduce a few definitions first: We denote by $\tilde{l}_{N,>}^{1, \omega}$ the largest and by $\tilde{l}_{N,>}^{2, \omega}$ the second largest length of all intervals $\left\{I_{N}^{j, \omega}\right\}_{j \in \mathbb{Z} \backslash\{0\}}$ without the two outer intervals

$$
I_{N}^{\mathrm{R}, \omega}:=I_{N}^{j_{N}^{\max , \omega}, \omega} \quad \text { where } \quad j_{N}^{\max , \omega}:=\max \left\{j \in \mathbb{Z}: I_{N}^{j, \omega}>0\right\},
$$

and

$$
I_{N}^{\mathrm{L}, \omega}:=I_{N}^{j_{N}^{\min , \omega}, \omega} \quad \text { where } \quad j_{N}^{\min , \omega}:=\min \left\{j \in \mathbb{Z}: I_{N}^{j, \omega}>0\right\} .
$$

as well as without $I_{N}^{0, \omega}$ for all $N \in \mathbb{N}$ and all $\omega \in \Omega$ for which they exist, that is, for which it is $\kappa_{N}^{\omega} \geqslant 4$; otherwise, i.e., if $\kappa_{N}^{\omega} \leqslant 4$, we set $\tilde{l}_{N,>}^{1, \omega}:=0$ and $\tilde{l}_{N,>}^{2, \omega}:=0$. Furthermore, we define the sets

$$
\begin{aligned}
& \Omega_{N}^{(\mathrm{R})}:=\left\{\omega \in \Omega:\left|I_{N}^{\mathrm{R}, \omega}\right| \leqslant \frac{1}{2 \nu} \ln (N)\right\}, \\
& \Omega_{N}^{(\mathrm{L})}:=\left\{\omega \in \Omega:\left|I_{N}^{\mathrm{L}, \omega}\right| \leqslant \frac{1}{2 \nu} \ln (N)\right\}, \\
& \Omega_{N}^{(0)}:=\left\{\omega \in \Omega:\left|I_{N}^{0, \omega}\right| \leqslant \frac{1}{2 \nu} \ln (N)\right\} .
\end{aligned}
$$

Note that for any $N \in \mathbb{N}$,

$$
\mathbb{P}\left(\Omega_{N}^{(\mathrm{R})}\right) \geqslant 1-\mathrm{e}^{-(1 / 2) \ln (N)},
$$




$$
\mathbb{P}\left(\Omega_{N}^{(\mathrm{L})}\right) \geqslant 1-\mathrm{e}^{-(1 / 2) \ln (N)},
$$

see, e.g., Kin93, Section 4.1], and

$$
\begin{aligned}
\mathbb{P}\left(\Omega_{N}^{(0)}\right) & \geqslant \mathbb{P}\left(\left\{\omega \in \Omega:\left|\left(0, \hat{x}_{1}(\omega)\right)\right| \leqslant \frac{1}{4 \nu} \ln (N)\right\} \cap\left\{\omega \in \Omega:\left|\left(\hat{x}_{0}(\omega), 0\right)\right| \leqslant \frac{1}{4 \nu} \ln (N)\right\}\right) \\
& \geqslant\left(1-\mathrm{e}^{-(1 / 4) \ln (N)}\right)+\left(1-\mathrm{e}^{-(1 / 4) \ln (N)}\right)-1=1-2 \mathrm{e}^{-(1 / 4) \ln (N)} .
\end{aligned}
$$

With Lemma A.5, with the fact that $\mathbb{P}\left(\lim \sup _{N \rightarrow \infty}\left(l_{N,>}^{1, \omega} / \ln (N)\right) \leqslant \kappa / \nu\right)=1$ for all $\kappa>2$, which can be shown in the same way as [KPS19b, Lemma A.2], and with Lemma A.6, where we choose $a=1$ and $\eta_{1}<\hat{\eta}<1 / 2$, we obtain

$$
\begin{aligned}
& \lim _{N \rightarrow \infty} \mathbb{P}\left(\left(\frac{\pi}{l_{N,>}^{2, \omega}}\right)^{2}-\left(\frac{\pi}{l_{N,>}^{1, \omega}}\right)^{2}>\frac{1}{N^{1-\eta_{1}}}\right) \\
& =\lim _{N \rightarrow \infty} \mathbb{P}\left(l_{N,>}^{1, \omega}-l_{N,>}^{2, \omega}>\frac{\pi^{-2}}{N^{1-\eta_{1}}} \cdot \frac{\left(l_{N,>}^{1, \omega} l_{N,>}^{2, \omega}\right)^{2}}{l_{N,>}^{1, \omega}+l_{N,>}^{2, \omega}}\right) \\
& \geqslant \lim _{N \rightarrow \infty} \mathbb{P}\left(l_{N,>}^{1, \omega}-l_{N,>}^{2, \omega}>\frac{\pi^{-2}}{N^{1-\eta}}\left(l_{N,>}^{1, \omega}\right)^{3}\right) \\
& \geqslant \lim _{N \rightarrow \infty} \mathbb{P}\left(\left\{\omega \in \Omega: l_{N,>}^{1, \omega}-l_{N,>}^{2, \omega}>\frac{\left(3 \nu^{-1} \ln (N)\right)^{3}}{\pi^{2} N^{1-\eta_{1}}}\right\}\right. \\
& \geqslant \lim _{N \rightarrow \infty} \mathbb{P}\left(\left\{\omega \in \Omega: \tilde{l}_{N,>}^{1, \omega}-\tilde{l}_{N,>}^{2, \omega}>\frac{\left(3 \nu^{-1} \ln (N)\right)^{3}}{\pi^{2} N^{1-\eta_{1}}}\right\}\right. \\
& \left.\quad \cap\left\{\omega \in \Omega: \frac{3}{4} \nu^{-1} \ln (N) \leqslant l_{N,>}^{1, \omega} \leqslant 3 \nu^{-1} \ln (N)\right\} \cap \Omega_{N}^{(0)} \cap \Omega_{N}^{(\mathrm{L})} \cap \Omega_{N}^{(\mathrm{R})}\right) \\
& \geqslant \lim _{N \rightarrow \infty} \mathbb{P}\left(\tilde{l}_{N,>}^{1, \omega}-\tilde{l}_{N,>}^{2, \omega}>\frac{1}{N^{1-\hat{\eta}}}\right) \\
& \quad \lim _{N \rightarrow \infty} \mathbb{P}\left(\left\{\frac{3}{4} \nu^{-1} \ln (N) \leqslant l_{N,>}^{1, \omega} \leqslant 3 \nu^{-1} \ln (N)\right\} \cap \Omega_{N}^{(0)} \cap \Omega_{N}^{(\mathrm{L})} \cap \Omega_{N}^{(\mathrm{R})}\right)-1 \\
& =1 .
\end{aligned}
$$

On the other hand, for the energy gap between the ground-state energy and the first excited energy on the largest interval we have

$$
\lim _{N \rightarrow \infty} \mathbb{P}\left(\left(\frac{2 \pi}{l_{N,>}^{1, \omega}}\right)^{2}-\left(\frac{\pi}{l_{N,>}^{1, \omega}}\right)^{2} \geqslant \frac{1}{3}\left(\frac{\nu \pi}{\ln (N)}\right)^{2}\right)=1,
$$

since $\lim _{N \rightarrow \infty} \mathbb{P}\left(l_{N,>}^{1, \omega} \leqslant(3 / \nu) \ln (N)\right)=1$. 
Finally, since the second eigenvalue $E_{N}^{2, \omega}$ is either the energy of the first excited state on the largest interval $l_{N,>}^{1, \omega}$ or the ground state energy of the second largest interval $l_{N,>}^{2, \omega}$, we obtain

$$
\begin{aligned}
& \lim _{N \rightarrow \infty} \mathbb{P}\left(\Omega_{N}^{1,1}\right) \\
& \quad \geqslant \lim _{N \rightarrow \infty} \mathbb{P}\left(E_{N}^{2, \omega}-E_{N}^{1, \omega}>\frac{1}{N^{1-\eta_{1}}}\right)+\lim _{N \rightarrow \infty} \mathbb{P}\left(E_{N}^{1, \omega} \leqslant\left[\left(1+\frac{\eta_{1}}{2}\right) \frac{\nu \pi}{\ln \left(L_{N}\right)}\right]^{2}\right)-1 \\
& =1 .
\end{aligned}
$$

Remark 3.2. Theorem 3.1 shows that, in probability, only the ground state is macroscopically occupied in the LS-model. We also recall that Corollary 2.10 shows that the ground state is $\mathbb{P}$-almost surely macroscopically occupied.

\section{A Miscellaneous results}

In this appendix we collect various results, some of which we referred to in the previous text. Lemma A.1 can be proved similarly as [KPS19b, Lemma A.7].

Lemma A.1. Under the assumptions of Theorem 2.5 one has, for $\rho>\rho_{c}(\beta)$ and given $\epsilon>0, \mathbb{P}$-almost surely

$$
\begin{aligned}
& \limsup _{N \rightarrow \infty} \int_{(\epsilon, \infty)} \mathcal{B}\left(E-\mu_{N}^{\omega}\right) \mathrm{d} \mathcal{N}_{N}^{\omega}(E) \leqslant \int_{(\epsilon, \infty)} \mathcal{B}(E) \mathrm{d} \mathcal{N}_{\infty}(E)+\frac{2}{\beta \epsilon} \mathcal{N}_{\infty}^{\mathrm{I}}(\epsilon), \\
& \liminf _{N \rightarrow \infty} \int_{(\epsilon, \infty)} \mathcal{B}\left(E-\mu_{N}^{\omega}\right) \mathrm{d} \mathcal{N}_{N}^{\omega}(E) \geqslant \int_{(\epsilon, \infty)} \mathcal{B}(E) \mathrm{d} \mathcal{N}_{\infty}(E)-\frac{4}{\beta \epsilon} \mathcal{N}_{\infty}^{\mathrm{I}}(2 \epsilon) .
\end{aligned}
$$

Lemma A.2. If the particle density $\rho$ is larger than the critical density, $\rho>\rho_{c}(\beta)$, then for any $c \in \mathbb{N}$ we $\mathbb{P}$-almost surely have

$$
\limsup _{N \rightarrow \infty} \frac{1}{N} \sum_{j=1}^{c} n_{N}^{j, \omega} \leqslant \frac{\rho_{0}(\beta)}{\rho} .
$$

Proof. Let $\epsilon>0$ be arbitrary. We recall the well-known fact that $\left(E_{N}^{j, \omega}\right)_{N \in \mathbb{N}}$ is, for every fixed $j \in \mathbb{N}$, a monotonically decreasing sequence. Hence, for all $1<j \leqslant c$ the sequence $\left(E_{N}^{j, \omega}\right)_{N \in \mathbb{N}}$ either converges to a constant $b_{j}>0$ or to zero. If the sequence does not converge to zero one obtains

$$
\lim _{N \rightarrow \infty} \frac{n_{N}^{j, \omega}}{N} \leqslant \beta^{-1} \lim _{N \rightarrow \infty} \frac{\left(E_{N}^{j, \omega}-\mu_{N}^{\omega}\right)^{-1}}{N} \leqslant \beta^{-1} \lim _{N \rightarrow \infty} \frac{2 b_{j}^{-1}}{N}=0
$$


where we used the fact that $\left(\mu_{N}^{\omega}\right)_{N \in \mathbb{N}}$ also converges to zero, see Theorem 2.5. Thus, in either case,

$$
\begin{aligned}
\limsup _{N \rightarrow \infty} \frac{1}{N} \sum_{j=1}^{c} n_{N}^{j, \omega} & \leqslant \limsup _{N \rightarrow \infty} \frac{1}{N} \sum_{j \in \mathbb{N}: E_{N}^{j, \omega} \leqslant \epsilon} n_{N}^{j, \omega} \\
& =\rho^{-1}\left[\rho-\liminf _{N \rightarrow \infty} \int_{(\epsilon, \infty)} \mathcal{B}\left(E-\mu_{N}^{\omega}\right) \mathrm{d} \mathcal{N}_{N}^{\omega}(E)\right] .
\end{aligned}
$$

Finally, with Lemma A.1 and taking $\inf _{\epsilon}$ on both sides we arrive at

$$
\limsup _{N \rightarrow \infty} \frac{1}{N} \sum_{j=1}^{c} n_{N}^{j, \omega} \leqslant \frac{\rho-\rho_{c}(\beta)}{\rho}=\frac{\rho_{0}(\beta)}{\rho},
$$

see also Remark 2.4.

In the rest of this appendix, we are concerned with the Luttinger-Sy model, see Section 3. Recall that $\left(\hat{x}_{j}(\omega)\right)_{j \in \mathbb{Z}}$ with $\ldots<\hat{x}_{-1}(\omega)<\hat{x}_{0}(\omega)<0<\hat{x}_{1}(\omega)<\hat{x}_{2}(\omega)<\ldots$ are the points generated by a Poisson point process of intensity $\nu>0$ and $\kappa_{N}^{\omega}$ is the number of Poisson points within the window $\left(-L_{N} / 2, L_{N} / 2\right)$. Furthermore, $\hat{l}^{j, \omega}=\mid\left(\hat{x}_{j}(\omega), \hat{x}_{j+1}(\omega) \mid\right.$ and $l_{N}^{j, \omega}=\left|\left(\hat{x}_{j}(\omega), \hat{x}_{j+1}(\omega)\right) \cap \Lambda_{N}\right|$. For the convenience of the reader, we present the next two lemmata with a proof, although more general versions of them can be found in [KPS19a, Appendix C].

Lemma A.3. For all $\epsilon>0$ and for $\mathbb{P}$-almost all $\omega \in \Omega$ there exists an $\tilde{N} \in \mathbb{N}$ such that for all $N \geqslant \tilde{N}$

$$
(1-\epsilon) \nu L_{N}<\kappa_{N}^{\omega}<(1+\epsilon) \nu L_{N}
$$

In particular, we $\mathbb{P}$-almost surely have

$$
\lim _{N \rightarrow \infty} \frac{\kappa_{N}^{\omega}}{L_{N}}=\nu .
$$

Proof. Firstly, we note that $1-\theta+\theta \ln (\theta)>0$ for any $\theta \in(0, \infty) \backslash\{1\}$ as well as that for arbitrary $N \in \mathbb{N}$,

$$
\mathbb{P}\left(\kappa_{N}^{\omega} \geqslant \theta \nu L_{N}\right) \leqslant \mathrm{e}^{-\nu L_{N}(1-\theta+\theta \ln \theta)}
$$

if $\theta \geqslant 1$ and

$$
\mathbb{P}\left(\kappa_{N}^{\omega} \leqslant \theta \nu L_{N}\right) \leqslant \mathrm{e}^{-\nu L_{N}(1-\theta+\theta \ln \theta)}
$$

for $0<\theta \leqslant 1$, see [SYZ12, Section 3.3.2]. The first part of this theorem now follows with the Borel-Cantelli lemma. 
Furthermore, using (A.3) we conclude

$$
\liminf _{N \rightarrow \infty} \frac{\kappa_{N}^{\omega}}{L_{N}} \geqslant(1-\epsilon) \nu \quad \text { and } \quad \limsup _{N \rightarrow \infty} \frac{\kappa_{N}^{\omega}}{L_{N}} \leqslant(1+\epsilon) \nu
$$

P-almost surely. Since $\epsilon>0$ can be chosen arbitrarily, the last statement of this theorem is also shown.

Lemma A.4. For all $0<\epsilon<1$ and for $\mathbb{P}$-almost all $\omega \in \Omega$ there exists an $\tilde{N} \in \mathbb{N}$ such that for all $N \geqslant \tilde{N}$,

$$
l_{N}^{j, \omega}\left\{\begin{array}{ll}
=\hat{l}^{j, \omega} & \text { if } j \in J_{\left[(1-\epsilon) \nu L_{N} / 2\right\rceil} \\
\leqslant \hat{l}^{j, \omega} & \text { if } j \in J_{\left\lfloor\nu L_{N}\right\rfloor} \\
=0 & \text { if } j \in \mathbb{Z} \backslash\left(J_{\left\lfloor\nu L_{N}\right\rfloor} \cup\{0\}\right)
\end{array},\right.
$$

where $J_{k}:=\{-k,-k+1, \ldots, k-1, k\} \backslash\{0\}, k \in \mathbb{N}$.

Proof. Recall that $I_{N}^{j, \omega}=\left(\hat{x}_{j}(\omega), \hat{x}_{j+1}(\omega)\right) \cap \Lambda_{N}$. Clearly, $l_{N}^{j, \omega} \leqslant \hat{l}^{j, \omega}$ for all $j \in \mathbb{Z}, N \in \mathbb{N}$, and $\omega \in \Omega$. In addition, we have $l_{N}^{j, \omega}=\hat{l}^{j, \omega}$ for all $j \in \mathbb{Z}, N \in \mathbb{N}$, and $\omega \in \Omega$ for which $I_{N}^{j, \omega} \subset \Lambda_{N}$, that is, for which $I_{N}^{j, \omega}$ is entirely within the window $\Lambda_{N}$. Similarly, we have $l_{N}^{j, \omega}=0$ for every $j \in \mathbb{Z}, N \in \mathbb{N}$, and $\omega \in \Omega$ for which $I_{N}^{j, \omega} \cap \Lambda_{N}=\varnothing$, that is, for which $I_{N}^{j, \omega}$ is entirely outside the window $\Lambda_{N}$.

We now determine when either of the last two cases holds. By $\kappa_{N}^{(1), \omega} \in \mathbb{N}$, we denote the number of atoms of the Poisson random measure within $\left(-L_{N} / 2,0\right]$. Similarly, the number of atoms of the Poisson random measure within $\left[0, L_{N} / 2\right)$ shall be $\kappa_{N}^{(2), \omega} \in \mathbb{N}$. Let an arbitrary $0<\epsilon<1$ be given. Since there are not $\kappa_{N}^{\omega}$ but $\kappa_{N}^{\omega}+1$ many intervals within $\Lambda_{N}$, we then pick an arbitrary $0<\epsilon^{\prime}<\epsilon$. With an appropriate version of Lemma A.3 we then conclude that for P-almost all $\omega \in \widetilde{\Omega}$ there exists an $\widetilde{N} \in \mathbb{N}$ such that for all $N \geqslant \widetilde{N}$,

$$
\frac{1}{2}\left(1-\epsilon^{\prime}\right) \nu L_{N} \leqslant \kappa_{N}^{(1), \omega} \leqslant \frac{1}{2}\left(1+\epsilon^{\prime}\right) \nu L_{N}
$$

and

$$
\frac{1}{2}\left(1-\epsilon^{\prime}\right) \nu L_{N} \leqslant \kappa_{N}^{(2), \omega} \leqslant \frac{1}{2}\left(1+\epsilon^{\prime}\right) \nu L_{N}
$$

Lemma A.5. For all $0<\epsilon<1$ there exists a set $\widetilde{\Omega} \subset \Omega$ with $\mathbb{P}(\widetilde{\Omega})=1$ and the following property: For every $\omega \in \widetilde{\Omega}$ there exists an $\tilde{N}=\tilde{N}(\epsilon, \omega)$ such that for all $N \geqslant \tilde{N}$ one has

$$
l_{N,>}^{1, \omega} \geqslant \nu^{-1}\left[\ln \left(L_{N}\right)-(1+\epsilon) \ln \left(\ln \left(L_{N}\right)\right)\right] .
$$

Proof. Let $0<\epsilon<1$ be arbitrarily given. As a first step, we conclude that

$$
\begin{aligned}
& \mathbb{P}\left(\max \left\{\hat{l}^{j, \omega}: j \in J_{\left[(1-\epsilon) \nu L_{N} / 2\right]}\right\}<\nu^{-1}\left[\ln \left(L_{N}\right)-(1+\epsilon) \ln \left[\ln \left(L_{N}\right)\right]\right]\right) \\
& \leqslant\left(1-\frac{\left[\ln \left(L_{N}\right)\right]^{1+\epsilon}}{L_{N}}\right)^{2\left[(1-\epsilon) \nu L_{N} / 2\right]}
\end{aligned}
$$


Here, we used the fact that $\left\{\hat{l}^{j, \omega}: j \in \mathbb{Z} \backslash\{0\}\right\}$ are mutually independent, exponentially distributed random variables. With the inequality $\ln (1-x) \leqslant-x$ for all $0<x<1$, we obtain the bound

$$
2\left\lceil\frac{(1-\epsilon) \nu L_{N}}{2}\right\rceil \cdot \ln \left[\left(1-\frac{\left[\ln \left(L_{N}\right)\right]^{1+\epsilon}}{L_{N}}\right)\right] \leqslant-(1-\epsilon) \nu\left[\ln \left(L_{N}\right)\right]^{1+\epsilon} \leqslant-2 \ln (N)
$$

for all but finitely many $N \in \mathbb{N}$. Therefore, we have

$$
\sum_{N=1}^{\infty}\left(1-\frac{\left[\ln \left(L_{N}\right)\right]^{1+\epsilon}}{L_{N}}\right)^{2\left[(1-\epsilon) \nu L_{N}\right] / 2}<\infty
$$

and, consequently, also

$$
\sum_{N=1}^{\infty} \mathbb{P}\left(\max \left\{\hat{l}^{j, \omega}: j \in J_{\left[(1-\epsilon) \nu L_{N} / 2\right]}\right\}<\nu^{-1}\left(\ln \left(L_{N}\right)-(1+\epsilon) \ln \left[\ln \left(L_{N}\right)\right]\right)\right)<\infty .
$$

By using Borel-Cantelli's lemma, it now follows that there exists a set $\widetilde{\Omega}_{1} \subset \Omega$ with $\mathbb{P}\left(\widetilde{\Omega}_{1}\right)=1$ and the following property. For every $\omega \in \widetilde{\Omega}_{1}$ there exists an $\widetilde{N}_{1}=\widetilde{N}_{1}(\epsilon, \omega) \in \mathbb{N}$ such that

$$
\max \left\{\hat{l}^{j, \omega}: j \in J_{\left[(1-\epsilon) \nu L_{N} / 2\right]}\right\} \geqslant \nu^{-1}\left\{\ln \left(L_{N}\right)-(1+\epsilon) \ln \left[\ln \left(L_{N}\right)\right]\right\}
$$

for all $N \geqslant \tilde{N}_{1}$.

On the other hand, according to Lemma A.4 there exists also a set $\widetilde{\Omega}_{2} \subset \Omega$ with $\mathbb{P}\left(\widetilde{\Omega}_{2}\right)=1$ and the following property. For every $\omega \in \widetilde{\Omega}_{2}$ there exists an $\widetilde{N}_{2}=\widetilde{N}_{2}(\epsilon, \omega) \in \mathbb{N}$ such that for all $N \geqslant \widetilde{N}_{2}$ it is

$$
\left\{\hat{l}^{j, \omega}: j \in J_{\left\lceil(1-\epsilon) \nu L_{N} / 2\right\rceil}\right\} \subset\left\{l_{N}^{j, \omega}: j \in \mathbb{Z} \backslash\{0\}\right\} \backslash\{0\} .
$$

and therefore

$$
\max \left\{\hat{l}^{j, \omega}: j \in J_{\left\lceil(1-\epsilon) \nu L_{N} / 2\right\rceil}\right\} \leqslant \max \left\{l_{N}^{j, \omega}: j \in \mathbb{Z} \backslash\{0\}\right\}=l_{N,>}^{1, \omega} .
$$

Altogether, we thus have shown that for every $\omega \in \widetilde{\Omega}_{1} \cap \widetilde{\Omega}_{2}$ there exists an $\tilde{N}:=$ $\max \left\{\widetilde{N}_{1}, \widetilde{N}_{2}\right\}$ such that for all $N \geqslant \widetilde{N}$ we have

$$
l_{N,>}^{1, \omega} \geqslant \max \left\{\hat{l}^{j, \omega}: j \in J_{\left\lceil(1-\epsilon) \nu L_{N} / 2\right\rceil}\right\} \geqslant \nu^{-1}\left\{\ln \left(L_{N}\right)-(1+\epsilon) \ln \left[\ln \left(L_{N}\right)\right]\right\} .
$$

Since $\mathbb{P}\left(\widetilde{\Omega}_{1} \cap \widetilde{\Omega}_{2}\right)=1$, we have proved this theorem.

For the next lemma recall the definition of $\tilde{l}_{N,>}^{1, \omega}$ and $\tilde{l}_{N,>}^{2, \omega}$ in the proof of Theorem 3.1 .

Lemma A.6. For any $a>0$ and $0<\hat{\eta}<1 / 2$, we have

$$
\lim _{N \rightarrow \infty} \mathbb{P}\left(\tilde{l}_{N,>}^{1, \omega}-\tilde{l}_{N,>}^{2, \omega}>\frac{a}{N^{1-\hat{\eta}}}\right)=1 .
$$


Proof. For all $k \in \mathbb{N}$, let $\left\{l^{j, \omega}\right\}_{j=1}^{k}$ be a set of $k$ independent and identically distributed random variables with common probability density $\nu \mathrm{e}^{-\nu l}$. For all $k \geqslant 2$, we define $l_{k,>}^{1, \omega}$ and $l_{k,>}^{2, \omega}$ as the largest and the second largest element of the set $\left\{l^{j, \omega}\right\}_{j=1}^{k}$, respectively. Then for any $2 \leqslant k \in \mathbb{N}$ and with

$$
\widetilde{\Omega}_{k}:=\left\{l_{k,>}^{1, \omega}-l_{k,>}^{2, \omega} \geqslant \frac{a}{(k+1)^{1-\hat{\eta}}}\right\}
$$

one has, see also [LZ07, Section 6.3],

$$
\begin{aligned}
\mathbb{P}\left(\widetilde{\Omega}_{k}\right) & =k(k-1) \int_{a(k+1)^{-1+\hat{\eta}}}^{\infty} \int_{0}^{x-a(k+1)^{-1+\hat{\eta}}}\left(1-e^{-\nu y}\right)^{k-2} \nu \mathrm{e}^{-\nu x} \nu \mathrm{e}^{-\nu y} \mathrm{~d} y \mathrm{~d} x \\
& =k \int_{a(k+1)^{-1+\hat{\eta}}}^{\infty}\left(1-\mathrm{e}^{-\nu\left(x-a(k+1)^{-1+\hat{\eta}}\right.}\right)^{k-1} \nu \mathrm{e}^{-\nu x} \mathrm{~d} x \\
& =\mathrm{e}^{-\nu a(k+1)^{-1+\hat{\eta}}} .
\end{aligned}
$$

We hence conclude that, see also [SYZ12, Section 3.3.3],

$$
\begin{aligned}
& \lim _{N \rightarrow \infty} \mathbb{P}\left(\tilde{l}_{N,>}^{1, \omega}-\tilde{l}_{N,>}^{2, \omega}>\frac{a}{\left(\kappa_{N}^{\omega}+1\right)^{1-\hat{\eta}}}\right) \\
& \geqslant \lim _{N \rightarrow \infty} \sum_{k=\left(1-L_{N}^{-\epsilon}\right) \nu L_{N}}^{\left(1+L_{N}^{-\epsilon}\right) \nu L_{N}} \mathbb{P}\left(\left\{l_{k-2,>}^{1, \omega}-l_{k-2,>}^{2, \omega}>\frac{a}{(k+1)^{1-\hat{\eta}}}\right\} \cap\left\{\kappa_{N}^{\omega}=k\right\}\right) \\
& \geqslant \lim _{N \rightarrow \infty} \sum_{k=\left(1-L_{N}^{-\epsilon}\right) \nu L_{N}}^{\left(1+L_{N}^{-\epsilon}\right) \nu L_{N}}\left[\mathbb{P}\left(\widetilde{\Omega}_{k-2}\right)+\mathbb{P}\left(\kappa_{N}^{\omega}=k\right)-1\right] \\
& =1
\end{aligned}
$$

where $\hat{\eta}<\epsilon<1 / 2$. Here, we used the fact that

$$
\lim _{N \rightarrow \infty} \sum_{k=\left(1-L_{N}^{-\epsilon}\right) \nu L_{N}}^{\left(1+L_{N}^{-\epsilon}\right) \nu L_{N}} \mathbb{P}\left(\kappa_{N}^{\omega}=k\right)=1 .
$$

since, see inequalities (A.4) and (A.5),

$$
\lim _{N \rightarrow \infty} \mathbb{P}\left(\kappa_{N}^{\omega}<\left(1-L_{N}^{-\epsilon}\right) \nu L_{N}\right)=\lim _{N \rightarrow \infty} \mathbb{P}\left(\kappa_{N}^{\omega}>\left(1+L_{N}^{-\epsilon}\right) \nu L_{N}\right)=0 .
$$


In addition,

$$
\begin{aligned}
\left|\lim _{N \rightarrow \infty} \sum_{k=\left(1-L_{N}^{-\epsilon}\right) \nu L_{N}}^{\left(1+L_{N}^{-\epsilon}\right) \nu L_{N}}\left[\mathbb{P}\left(\widetilde{\Omega}_{k-2}\right)-1\right]\right| \leqslant & \lim _{N \rightarrow \infty} \sum_{k=\left(1-L_{N}^{-\epsilon}\right) \nu L_{N}}^{\left(1+L_{N}^{-\epsilon}\right) \nu L_{N}}\left|\mathbb{P}\left(\widetilde{\Omega}_{k-2}\right)-1\right| \\
& \leqslant \lim _{N \rightarrow \infty} \sum_{k=\left(1-L_{N}^{-\epsilon}\right) \nu L_{N}}^{\left(1+L_{N}^{-\epsilon}\right) \nu L_{N}} \nu a(k-1)^{-1+\hat{\eta}} \\
& \leqslant \lim _{N \rightarrow \infty} \nu a \frac{2 L_{N}^{-\epsilon} \nu L_{N}}{\left[\left(1-L_{N}^{-\epsilon}\right) \nu L_{N}-1\right]^{1-\hat{\eta}}} \\
& \leqslant \lim _{N \rightarrow \infty} \nu a \frac{2 \nu L_{N}^{1-\epsilon}}{\left(\frac{1}{2} \nu L_{N}\right)^{1-\hat{\eta}}} \\
& =0,
\end{aligned}
$$

since $\hat{\eta}<\epsilon$. Lastly, again due to inequality (A.5),

$$
\begin{aligned}
& \lim _{N \rightarrow \infty} \mathbb{P}\left(\tilde{l}_{N,>}^{1, \omega}-\tilde{l}_{N,>}^{2, \omega}>\frac{a}{\left(2 \nu \rho^{-1} N\right)^{1-\hat{\eta}}}\right) \\
& \quad \geqslant \lim _{N \rightarrow \infty} \mathbb{P}\left(\left\{\tilde{l}_{N,>}^{1, \omega}-\tilde{l}_{N,>}^{2, \omega}>\frac{a}{\left(\kappa_{N}^{\omega}+1\right)^{1-\hat{\eta}}}\right\} \cap\left\{\kappa_{N}^{\omega} \leqslant 2 \nu \rho^{-1} N-1\right\}\right) \\
& \quad \geqslant \lim _{N \rightarrow \infty} \mathbb{P}\left(\tilde{l}_{N,>}^{1, \omega}-\tilde{l}_{N,>}^{2, \omega}>\frac{a}{\left(\kappa_{N}^{\omega}+1\right)^{1-\hat{\eta}}}\right)+\lim _{N \rightarrow \infty} \mathbb{P}\left(\kappa_{N}^{\omega} \leqslant 2 \nu \rho^{-1} N-1\right)-1 \\
& =1 .
\end{aligned}
$$




\section{References}

[Bau01] H. Bauer, Measure and integration theory, de Gruyter, 2001.

[Ber83] M. van den Berg, On condensation in the free-boson gas and the spectrum of the Laplacian, J. Stat. Phys. 31 (1983), 623-637.

[BL82] M. van den Berg and J. T. Lewis, On generalized condensation in the free boson gas, Physica A: Statistical Mechanics and its Applications 110 (1982), 550-564.

[BLL86] M. van den Berg, J. T. Lewis, and M. Lunn, On the general theory of Bose-Einstein condensation and the state of the free boson gas, Helvetica Physica Acta 59 (1986), $1289-1310$.

[BLP86] M. van den Berg, J. T. Lewis, and J. V. Pulé, A general theory of Bose-Einstein condensation, Helvetica Physica Acta 59 (1986), 1271-1288.

[Egg72] T. P. Eggarter, Some exact results on electron energy levels in certain one-dimensional random potentials, Phys. Rev. B 5 (1972), 3863-3865.

[FP92] A. Figotin and L. A. Pastur, Spectra of random and almost-periodic operators, Springer, 1992.

[GHK05] F. Germinet, P. D. Hislop, and A. Klein, On localization for the Schrödinger operator with a Poisson random potential, Comptes Rendus Mathematique 341 (2005), 525528.

[GHK07] _ Localization for Schrödinger operators with Poisson random potential, J. Eur. Math. Soc. 9 (2007), 577-607.

[HS65] E. Hewitt and K. Stromberg, Real and abstract analysis: a modern treatment of the theory of functions of a real variable, Springer, 1965.

[Kin93] J. F. C. Kingman, Poisson processes, Clarendon Press, 1993.

[KL73] M. Kac and J. M. Luttinger, Bose-Einstein condensation in the presence of impurities, J. Math. Phys. 14 (1973), 1626-1628.

[KL74] _ Bose-Einstein condensation in the presence of impurities. II, J. Math. Phys. 15 (1974), 183-186.

[KPS19a] J. Kerner, M. Pechmann, and W. Spitzer, Bose-Einstein condensation in the Luttinger-Sy model with contact interaction, Ann. Henri Poincaré 20 (2019), 21012134.

[KPS19b] _ On Bose-Einstein condensation in the Luttinger-Sy model with finite interaction strength, J. Stat. Phys. 174 (2019), 1346-1371.

[LMW03] H. Leschke, P. Müller, and S. Warzel, A survey of rigorous results on random Schrödinger operators for amorphous solids, Markov Processes and Related Fields 9 (2003), 729-760. 
[LPZ04] O. Lenoble, L. A. Pastur, and V. A. Zagrebnov, Bose-Einstein condensation in random potentials, Comptes Rendus Physique 5 (2004), 129-142.

[LS73a] J. M. Luttinger and H. K. Sy, Bose-Einstein Condensation in a one-dimensional model with random impurities, Phys. Rev. A 7 (1973), 712-720.

[LS73b] Low-lying energy spectrum of a one-dimensional disordered system, Phys. Rev. A 7 (1973), 701-712.

[LZ07] O. Lenoble and V. A. Zagrebnov, Bose-Einstein condensation in the Luttinger-Sy model, Mark. Proc. Rel. Fields 13 (2007), 441-468.

[Sto95] G. Stolz, Localization for random Schrödinger operators with Poisson potential, Annales de l'IHP Physique théorique 63 (1995), 297-314.

[SW16] R. Seiringer and S. Warzel, Decay of correlations and absence of superfluidity in the disordered Tonks-Girardeau gas, New Journal of Physics 18 (2016), no. 3, 035002.

[SYZ12] R. Seiringer, J. Yngvason, and V. A. Zagrebnov, Disordered Bose-Einstein condensates with interaction in one dimension, J. Stat. Mech.: Theory and Experiment 2012 (2012), P11007.

[Szn98] A.-S. Sznitman, Brownian motion, obstacles and random media, Springer, 1998. 